\title{
Prevalence of $H E R 2$ Expression and Its Correlation with Clinicopathological Parameters in Gastric or Gastroesophageal Junction Adenocarcinoma in North-East Indian Population
}

\author{
Partha S Roy', Tomar Nyodu ${ }^{1}$, Munlima Hazarika ${ }^{1 *}$, B J Saikia ${ }^{1}$, C Bhuyan', \\ Amit Inamdar', C W Nyuthe ${ }^{1}$, B Borthakur ${ }^{2}$, J D Sharma ${ }^{3}$
}

\begin{abstract}
Objective: Human epidermal growth factor receptor 2 (erbb2/HER2) overexpression, has now been implicated in advanced gastric and gastroesophageal junction cancers. The study was conducted to determine the rate of HER 2 positivity in patients with locally advanced or metastatic gastric and gastroesophageal adenocarcinoma in North-East India and to assess the impact of various demographic and clinical parameters on HER2 positivity. Methods: A total of 68 patients of age $>18$ years of gastric and gastroesophageal adenocarcinoma diagnosed on histopathological examination from September 2016 to February 2018 at Dr B Borooah Cancer Institute, Assam were enrolled for the observational (epidemiological) study. All patients were subjected to the HER2 immunohistochemistry test using a FDA-approved, standardized test kit. HER 2 expression was correlated with various demographic and clinicopathological parameters. Results: The overall rate of HER2 positivity in the population studied was $56 \%(\mathrm{n}=38)$. The rate was non-significantly higher in male, older age group ( $>60$ years) and Hindu population. Similarly, HER 2 positivity rate was higher in patients with well differentiated histology and was more common in patients with stage II and III diseases, but neither of the associations is statistically significant. HER2 positivity rate was significantly higher in proximal and in GEJ tumours ( $56 \%$ versus $44 \%, \mathrm{P}=0.002$ ). Conclusion: HER 2 overexpression was evident in $56 \%$ of the North-East Indian patients with locally advanced and metastatic gastric and gastroesophageal adenocarcinoma. The overexpression correlated significantly with primary tumour site. Routine testing of gastric and gastroesophageal tumours for HER 2 expression is recommended to provide a therapeutic advantage in Indian patients.
\end{abstract}

Keywords: Gastric cancer- gastroesophageal adenocarcinoma- human epidermal factor receptor- immunohistochemistry

Asian Pac J Cancer Prev, 20 (4), 1139-1145

\section{Introduction}

Gastric cancer is a leading health-care problem worldwide and is the fifth most common cancer in the world, with 952,000 new cases diagnosed in 2012 Worldwide 0.7 million people die of gastric cancer each year (accounts for $8.8 \%$ of all cancer-related deaths) [GLOBACON, 2012]. Gastric cancer is the second most common cancer among men and third-most among females in Asia (Rahman et al., 2014) and is the second most common cause of cancer-related deaths in India (Dikshit et al., 2012). A high incidence of gastric cancer has been reported from Southeast Asia, most commonly from Japan, China and South Korea (Ferlay et al., 2012).

The age-adjusted rate (AAR) of gastric cancer among urban registries in India is (3.0-13.2) compared to the worldwide AAR (4.1-95.5) with China having the highest incidence of AAR. Amongst all the population based cancer registries in India, Papumpure district (Arunachal
Pradesh, India) has the highest AAR of 50.2\% followed by Aizawl district (Mizoram, India) with AAR of $43.9 \%$ both in males and females (ICMR 2016). The long term ingestion of high concentration of nitrates found in dried, smoked and salted foods appears to be associated with a higher risk in this part of the world.

In advanced stage disease, the 5-year survival is less than $30 \%$ in developed countries and is around $20 \%$ in developing countries (Kang et al., 2010). Surgery is the mainstay of treatment in early stage disease. Although chemotherapy improves survival in patients with advanced gastric cancer, there is no globally accepted standard first-line regimen. Even with optimal combination chemotherapy, the median survival in Western studies remains less than 1 year in advanced settings (Van Cutsem et al., 2006; Cunningham et al., 2008). Attention has turned to potential molecular targets in gastric and gastroesophageal junction (GEJ) adenocarcinomas.

Human epidermal growth factor receptor 2 (HER2)

${ }^{1}$ Department of Medical Oncology, ${ }^{2}$ Department of Surgical Oncology, ${ }^{3}$ Department of Pathology, Dr B Borooah Cancer Institute, Guwahati, Assam, India.*For Correspondence: drmunlimahazarika@yahoo.com 
is a $185-\mathrm{kDa}$ trans-membrane tyrosine kinase receptor of the HER family and is a proto-oncogene encoded by erb2 on chromosome 17. HER2 gene amplification and protein overexpression play an important role in the proliferation, apoptosis, adhesion, angiogenesis and aggressiveness of many solid tumours. Although HER2 expression was initially associated with breast cancer it has now been implicated in various cancers including, colon, bladder, ovary, uterine cervix, gastric and GEJ cancers (Reicheelt et al., 2007; Kaur et al., 2011).

HER 2 overexpression in gastric cancer IHC was first described in 1986 (Gravaalos et al., 2008). Large numbers of studies on HER 2 positivity in gastric cancer have been published from Western world, relating it to important clinicopathological characteristics and survival. HER2 positivity in gastric cancer ranges from as low as $4 \%$ to as high as 53\% (Kunz et al., 2012; Shan et al., 2013; Abrahao-Machad et al., 2016; Gharsalli et al., 2017). In a literature review of a large population involving more than 8000 patients, median HER 2 positivity of $20.2 \%$ was observed (Maressch et al., 2012). The HER2 expression rate in Asians may be higher than that in Europeans (Lei et al., 2017). In an Egyptian population of 76 resectable gastric carcinoma patients, the rate of HER2/neu positivity was found to be high (about 54\%) (Abdel-Salam et al., 2018).

Routinely one paraffin block is used for HER2/neu assessment by IHC. Using two paraffin blocks to assess HER2/neu expression may identify more patients with HER2/neu positive gastric cancer, by reducing false-negative rate (Xiaowen et al., 2015).

HER2 is a well-established therapeutic target in breast cancer. HER 2 overexpression in metastatic gastric cancer patients may be a predictor of poor prognosis (Aguair Junior et al., 2016). Addition of trastuzumab to chemotherapy has been shown to improve the survival in patients with HER2-positive gastric and GEJ carcinoma in Trastuzumab for Gastric Cancer (ToGA) trial (Bang et al., 2010). In 2010 trastuzumab received approval for the use in combination with 5-flurouracil (5-FU) or capecitabine plus cislatin for the first-line treatment of patients with HER 2 positive metastatic adenocarcinoma of the gastric or GEJ after the result of the phase III ToGA trial (Bilici et al., 2014). Median overall survival was significantly improved with trastuzumab plus chemotherapy compared to chemotherapy alone: 13.8 (12.0-16.0) months versus 11.1 (10.0-13.0) months, respectively $(\mathrm{P}=0.0046)$ (Bang et al., 2010).

Studies conducted so far suggest the need for optimizing HER 2 testing as appropriate interpretation for these test results could translate into delivery of optimal therapy. It has been reported that only patients with high levels of HER 2 expression derive maximum benefit from trastuzumab based therapy (Ruschoff et al., 2012).

In the year 2015, India reported a total of 63,043 gastric cancer cases, with 3,806 cases from North-East India. Dr B Borooah Cancer Institute, a regional cancer centre of the North-East India recorded 320 cases of gastric adenocarcinoma in 2015 (ICMR, 2016).

However, studies on the frequency of HER2-positivity among the patients in the North-East region of the
India are limited. Therefore, this study was conducted to evaluate the prevalence of HER2 in gastric and GEJ adenocarcinoma in North-East Indian population and to correlate HER2 overexpression with clinicopathological parameters.

\section{Materials and Methods}

It was an observational study (epidemiological study) wherein the data were collected in a prospective manner. A total of 340 cases of gastric and GEJ adenocarcinoma diagnosed on biopsy specimen in the pathology department of Dr B Borooah Cancer Institute (BBCI) over a time span of one and half years (September 2016 to February 2018) were included in the study. The study received the approval from the Institutional Ethics Committee (BBCI/M-119/ MEC/2195/2016).

The study included men and women over 18 years of age, newly diagnosed with locally advanced or metastatic histopathologically confirmed gastric/GEJ adenocarcinoma. Patients aged less than 18 years and patients who received chemotherapy and/or radiotherapy earlier were excluded from the study.

The primary objective of the study was to determine the incidence of HER2 positivity in patients with locally advanced or metastatic gastric/GEJ adenocarcinoma in North-East India. The secondary objective was to evaluate correlation of HER2 overexpression with demographic and clinicopathological parameters.

The pathology records of all patients diagnosed with gastric/GEJ adenocarcinoma at BBCI were obtained electronically from September 2016 to February 2018 and investigations for HER2 status were included in the study. Among 340 histopathologically confirmed cases of gastric/GEJ adenocarcinoma, 68 cases that were subjected to further HER2 immunohistochemistry (IHC) test were included for the final analysis.

Clinical data, including patient's demographic profile and pathological parameters namely, tumour site, stage and histological grade were recorded.

HER 2 overexpression was determined by IHC test using an FDA-approved, standardised test kit (Hercep test kit ${ }^{\mathrm{TM}}$, DakoCytomation Denmark A/ $\mathrm{S}^{\mathrm{TM}}$, Glostrup, Denmark). For determination of HER2 expression, a semi-quantitative scoring criterion was applied in sections and the results were recorded as positive, negative and equivocal according to the staining patterns (Hofmann et al., 2008; Abrahao-Machad et al., 2016). (Table 1) Scores of $3+$ was taken as positive. Score values of 0 and $1+$ were taken as negative. HER2 IHC score $2+$ were considered as equivocal and should be further tested for gene amplification by fluorescence in-situ hybridization (FISH) method. Confirmation of cases reported as equivocal (HER2 IHC score $2+$ ) by FISH could not be done in our study due to financial restraints. Therefore, score 2+ was also included as negative for all practical purposes. Overall HER 2 overexpression in gastric and GEJ adenocarcinoma was evaluated and was further correlated with various clinicopathological features. Impact of trastuzumab on survival in HER2-positive cases was not studies.

Data that are collected was checked for consistency 
and completeness, and entered in data base software. Summarization and analysis was carried out using descriptive statistics. Statistical analysis was done with SPSS version 16.0 software. Categorical variables were expressed as frequencies and percentages. Nominal categorical data between the groups were compared using Chi-square test $\left(\chi^{2}\right)$. Survival analysis was done using Kaplan-Meier (log-rank tests) survival curves. For all statistical tests, a $\mathrm{P}<0.05$ was considered as statistically significant (at 95\% CI).

\section{Results}

A total of 68 cases were included for the study. Clinicopathological characteristics and their association with HER2 final status and IHC scores are shown in Table 2.

\section{Patient demographics and tumour characteristics}

Patients age at diagnosis ranged from 32 to 80 years (mean age of presentation was 58 years). Of total 68 patients, $40(59 \%)$ were male and $28(41 \%)$ were female (male to female ratio was $1.4: 1$ ). The most common age group was 40 to 60 years $(54.4 \%)$ followed by age group of more than 60 years $(38 \%)$. The patient's age less than 40 years constitutes only $7 \%$. Majority of the patients are from Hindu community (54\%) followed by Muslim community $(37 \%)$. The location of the tumour was at the GEJ in $20.5 \%$, proximal part of stomach (excluding GEJ) in $17.6 \%$, and body/distal part of stomach in $61.7 \%$ patients. Poorly differentiated tumours were observed in $25(36.7 \%)$, moderately differentiated tumour in 20 (29.4\%), and well differentiated tumours in $23(33.8 \%)$ of patients.

HER 2 overexpression of $3+$ was considered as positive; whereas score values of $2+, 1+$ and 0 were taken as negative. Of the total 68 cases enrolled the study, $56 \%$ $(\mathrm{n}=38)$ tested positive for HER2 by IHC (score $3+$ ) and $44 \%(\mathrm{n}=30)$ were negative for HER2 (score $2+, 1+$ and $0)$ by IHC.

The median age of presentation is 57.5 years in HER2 positive cases and is 58 years in HER2 negative cases. The maximum number of HER 2 positivity tumours was detected in patients aged more than 40 years (40 to 60 years age group). None of the patients in the IHC3+ category were younger than 32 years of age and the oldest patient in the series was an 80 year old man, whereas the youngest age was 35 years in HER2 negative group.
The rate of HER 2 positivity was higher in men $(66 \%$ versus $34 \%$ ) than women, but there was no association between HER 2 expression and patient's gender.

\section{Association/correlation of HER2 status with clinicopathological features}

There was no statistically significant correlation between HER 2 positivity with age, gender, race, and tumour staging.

\section{HER2 positivity rate and gastric cancer site}

Tumour located in different parts of the stomach such as the fundus, lesser curvature, body, antrum and pylorus were grouped as gastric body tumours, whereas tumour located in the cardia, lower end of esophagus, GEJ, proximal stomach, and cardio-esophageal junction were grouped as GEC (GEJ cancers) tumours. The incidence of HER2 positivity in GEC $(55 \%)$ was higher than that in gastric body (45\%); this association was found to be statistically significant $(\mathrm{P}=0.006)$. The overall HER2 positivity rate for patients characterised as having a gastric site (proximal and distal stomach combined) was $79 \%$ and $21 \%$ for those with GEJ site, where the association was found to highly significant $(\mathrm{P}=0.002)$.

Tumours arising in the distal stomach accounted for $45 \%$ of IHC3 + cases; $20.5 \%$ were located in the in the GEJ and another 34\% located in the proximal stomach (excluding GEJ), where the variation seen to be statistically significant $(\mathrm{P}=0.002)$. In fact, this is the first report of HER2 positivity status in pure GEJ tumours, where GEJ tumours accounts for $20.5 \%(n=14)$ of total 68 case. When all the proximal tumours are combined (that also includes GEJ tumours), HER2 positivity seen in $55 \%$ of cases (variation is significant with $\mathrm{P}=0.006$ ).

\section{HER 2 positivity rate and tumour grade/tumour differentiation}

Our study showed a non-significant association between HER 2 expression and well differentiated histology. The HER2 positivity rate for tumour grade was $50 \%$ for well differentiated, $24 \%$ for poorly differentiated, and $26 \%$ for moderately differentiated $(\mathrm{P}=0.051)$. Studies have shown both an association and non-association between HER 2 overexpression and tumour differentiation. This discrepancy may be attributed to varying sample sizes and lower prevalence of HER2 in gastric/gastroesophageal cancers.

HER2 negative carcinomas with HER2 IHC score 1+

Table 1. Human Epidermal Growth Factor Receptor 2 Immunohistochemistry Scoring Criteria for Gastric Cancer (Hofmann et al.)

\begin{tabular}{lcc}
\hline Staining pattern & IHC score & HER2 overexpression \\
\hline No reactivity or membranous reactivity in $<10 \%$ of tumour cells & 0 & Negative \\
$\begin{array}{l}\text { Faint or barely perceptible reactivity in } \geq 10 \% \text { of tumour cells; cells are reactive only in } \\
\text { part of their membrane }\end{array}$ & $1+$ & Negative \\
$\begin{array}{l}\text { Weak to moderate, complete, basolateral, or lateral membranous reactivity in } \geq 10 \% \text { of } \\
\text { tumour cells }\end{array}$ & $2+$ & Equivocal $^{*}$ \\
Strong, complete, basolateral, or lateral membranous reactivity in $\geq 10 \%$ of tumour cells & $3+$ & Positive \\
\hline
\end{tabular}

*Equivocal HER2 expression by IHC to be confirmed by FISH 
Table 2. Association between HER2 Status and Clinicopathological Parameters

\begin{tabular}{|c|c|c|c|c|}
\hline & Overall $n=68(\%)$ & HER2 positive $\mathrm{n}=38(55.8 \%)$ & HER2 negative $n=30(44.1 \%)$ & $P$ value \\
\hline Age Group (years) & & & & 0.09 \\
\hline$<40$ & $5(7.3)$ & $3(7.8)$ & $2(6.6)$ & \\
\hline $40-60$ & $27(54.4)$ & $21(55.0)$ & $16(53.3)$ & \\
\hline$>60$ & $26(38.2)$ & $14(36.8)$ & $12(40.0)$ & \\
\hline Gender & & & & 0.189 \\
\hline Male & $40(58.8)$ & $25(65.7)$ & $15(50.0)$ & \\
\hline Female & $28(41.1)$ & $13(34.2)$ & $15(50.0)$ & \\
\hline Race & & & & 0.094 \\
\hline Hindu & $37(54.4)$ & $25(65.7)$ & $12(40.0)$ & \\
\hline Muslim & $25(36.7)$ & $11(28.9)$ & $14(46.6)$ & \\
\hline Christian & $6(8.8)$ & $2(5.2)$ & $4(13.3)$ & \\
\hline Tumour Location & & & & 0.002 \\
\hline GEJ & $14(20.5)$ & $8(21.0)$ & $6(20.0)$ & \\
\hline Proximal (excluding GEJ) & $12(17.6)$ & $13(34.2)$ & $0(-)$ & \\
\hline Body/distal gastric & $42(61.7)$ & $17(44.7)$ & $24(80.0)$ & \\
\hline Histological Grade & & & & 0.051 \\
\hline Well differentiated & $23(33.8)$ & $19(50.0)$ & $4(13.3)$ & \\
\hline Moderately differentiated & $20(29.4)$ & $10(26.3)$ & $10(33.3)$ & \\
\hline Poorly differentiated & $25(36.7)$ & $9(23.6)$ & $14(53.3)$ & \\
\hline TNM Staging (clinical) & & & & 0.312 \\
\hline Stage II & $10(14.7)$ & $6(15.7)$ & $4(13.3)$ & \\
\hline Stage III & $29(42.6)$ & $16(42.1)$ & $13(43.3)$ & \\
\hline Stage IV & $22(32.3)$ & $10(26.3)$ & $12(40.0)$ & \\
\hline NK & $7(10.2)$ & $6(15.7)$ & $1(3.3)$ & \\
\hline
\end{tabular}

and 0 aggregated all tumours with poorly differentiated histological grades. Poorly differentiated histology accounts for $47 \%$ of all HER 2 negative carcinomas (score $2+, 1+$ and 0 ), whereas, poorly differentiated histology is seen in only $29 \%$ of $H E R 2$ positive tumours. $H E R 2$ positive tumours are more associated with well differentiated histology (in $50 \%$ cases versus in $20 \%$ of HER 2 negative cases); however, the association is statistically not significant $(\mathrm{P}=0.051)$.

\section{HER 2 positivity rate and TNM staging}

HER2-positivity was more commonly found to be associated with stage III diseases (16\% in stage II, $42 \%$ in stage III and $26 \%$ in stage IV). However, no significant association was observed between HER 2 expression and TNM staging.

Statistical significance of HER2 positivity rates between subgroups

The chi-squared $\left(\chi^{2}\right)$ test was used to assess the statistical significance of HER2 positivity rates across the different demographic parameters for the following subgroups: gastric cancer site, age and tumour grade. For all analyses done, the $\left(\chi^{2}\right)$ test was significant, for the association between tumour location and HER2 expression concluding that there was at least one significant difference between the HER 2 positive rates and the demographic parameters of the individual subgroups.

\section{Discussion}

HER2 expression in gastric/gastroesophageal cancers has been known for over years. In addition to being implicated in the pathogenesis of cancers, HER2 has also been evaluated as a therapeutic target. Efficacy of trastuzumab in patients with breast cancer has led to emerging interest in its antitumor activity in patients with HER2 positive gastric carcinoma. Many studies have investigated the role of trastuzumab in the management of gastric/gastroesophageal cancer. The implication of HER 2 overexpression on prognosis remains unknown in patients with metastatic gastric cancer who do not receive trastuzumab.

Our study describes findings from the clinical audit of HER2 expression in gastric cancer in North-East India centre, providing the first insight into the rate of HER2-positivity in gastric and gastroesophageal adenocarcinoma. Based on an analysis of 340 cases of gastric/gastroesophageal cancer biopsy specimens, 68 cases were finally analysed for HER 2 expression by IHC. An overall HER 2 positivity rate of $56 \%$ was observed in our study. The rate is higher compared to the range reported in the literature from different populations (ranges from as low as $4.4 \%$ to $53.4 \%$ ) (Abdel-Salam et al., 2018).

In our study, HER 2 overexpression was observed in $56 \%$ cases of gastric or gastroesophageal adenocarcinoma 
which is the highest positivity rate reported till date. Variation in HER2-positivity rates between countries and different populations may be explained in part by the proportion of carcinomas with certain characteristics with which HER2-positivity has been correlated. HER2-positivity rates were higher in the proximal gastric cancer site than in the gastric site $(55 \%$ versus $44 \%$ ), a correlation that has been consistently reported in the literature (Shan et al., 2013; Van et al., 2015). Higher HER2 expression was seen in older age, males and in Hindu population, however, the correlation was not statistically significant. Similarly, in an Iranian population, older age and male sex appears to be more associated with HER2/neu gene expression (Azarhoosh et al., 2017). A non-significant association was also noted between HER 2 overexpression and stage II/III gastric/ gastroesophageal cancers.

HER2 overexpression was correlated with the various clinicopathological parameters in gastric cancer patients: male sex, proximally located tumour, advanced stage, lymph node metastasis, distant metastasis, poorly differentiated tumour, and intestinal subtype (Lei et al., 2017). However, in a small case series from Pakistan, HER2/neu was significantly expressed in low grade gastric cancer, predominantly seen in females, age $>60$ years, intestinal subtype, and stage IIIC tumours (Shabbir et al., 2018).

HER2-positivity was associated with intestinal subtype ( $\mathrm{P}=0.048)$, well to moderately differentiated tumour $(\mathrm{P}=0.004)$ and presence of lymphovascular invasion ( $\mathrm{P}=0.031$ ) (Renato et al., 2015). In our study we did not see any significant correlation between $H E R 2$ overexpression and histological grades of tumour, although a trend towards significance is observed with well differentiated histology being more commonly seen in HER2 positive cases $(\mathrm{P}=0.051)$. However, HER $2 \mathrm{IHC}$ score $1+$ and 0 were exclusively seen to be associated with poorly differentiated histology.

Consistent with previous reports, HER2-positivity was more frequent with well- or moderately differentiated histology (50\% and 26\%, respectively) (Shan et al., 2013; Son et al., 2014; Rajagopal et at., 2015), compared with poorly differentiated carcinomas (23\%). In our study, HER2/neu gene expression is more commonly seen in stage III disease ( $42 \%$ in stage III versus $26 \%$ in stage IV), unlike other studies where HER2/neu gene expression is significantly more seen in stage IV of gastric cancer with a larger tumour mass, that can be partly explained by slightly higher numbers of patients with stage III disease (Azarhoosh et al., 2017). A study from South Asian population also found significantly higher HER2/neu expression in low grade tumours and stage III tumours (Shabbir et al., 2018).

Although, many authors did not report any significant association between site of tumour and HER2-positivity, the conflicting results have been reported regarding tumour location and HER 2 expression. In our study proximally located tumours constitutes $38 \%$ of cases which may be the reason for higher HER2 positivity rate of $55 \%(\mathrm{P}=0.006)$. GEJ adenocarcinoma alone accounts for $21 \%(\mathrm{n}=14$ ) of all cases (with HER2 positivity of $21 \%$ ); proximal tumours other than GEJ tumours accounts for $18 \%$ (HER2 positivity of $34 \%$ ), which is highly significant $(\mathrm{P}=0.002)$. Fan et al., (2013) has also reported a significant association of $H E R 2$ positivity with proximal tumours. In fact, this is the first report of HER2 positivity status in pure GEJ adenocarcinoma. Another reason for high HER2 positivity rate may be because of different demographic features of the North-East part of the India as compared to other part of the World. There was no previous similar study available to compare our study in the North-East part of the India.

A number of limitations of this study warrant mention. Most notably, a considerable amount of patient data were not collected particularly on tumour stage and gender, preventing further analysis of these parameters. The main weakness of our study was the non-availability of FISH/ISH analysis to determine HER2 amplification for HER2 equivocal (score 2+) cases, which could have probably influenced the variations/associations amongst the different clinicopathological parameters. Although the IHC continues to play an essential role in HER2 status assessment, the overall reliability of HER 2 evaluation by IHC, however, can be affected by diverse pre-analytical, analytical and post-analytical variables.

The high proportion of equivocal (IHC 2+) HER2 cases is also a concern. Many patients in Asian countries are not referred for confirmatory FISH/ISH testing, primarily due to the high cost of these assays and HER2 targeted therapy should a positive result be found.

Given these limitations it is probably unwise to consider these results as a wholly accurate representation of the incidence of HER2-positivity in gastric/gastroesophageal cancer in North-East India. A well conducted prospective randomized trial probably can give us the exact prevalence of HER2 positivity in this unique geographical area of North-East India.

In conclusion, this study highlights the need of further defining the role of HER2 expression in gastric/ gastroesophageal adenocarcinoma in North-East Indian patients. Given the significant burden of gastric cancer in this region, these data are important to increase our understanding and awareness of the disease. Increased education and awareness of HER2 testing in gastric/ gastroesophageal cancer, as well as improved early detection are what is needed to improve outcomes. Routine testing of gastric and gastroesophageal tumours for HER2 expression is recommended to provide a therapeutic advantage in Indian patients.

\section{Funding source}

None.

\section{Conflict of interest}

The authors have no conflicts of interest to declare.

\section{Acknowledgements}

The authors acknowledge their gratitude towards Hospital Based Cancer Registry (HBCR) of Dr. B Borooah Cancer Institute, Guwahati, Assam for providing data.

Asian Pacific Journal of Cancer Prevention, Vol 201143 


\section{References}

Abdel-Salam RA, El-Hawary A, Mohamed MA, Gamil T (2018). Immunohistochemical expression of Her2/neu in gastric carcinomas in Egyptian patients. J Clin Pathol Diagn, 1, 1-6.

Abrahao-Machado LF, Scapulatempo-Neto C (2016). HER2 testing in gastric cancer: An update. World J Gastroenterol, 22, 4619-25.

Aguir Junior PN, Artigiani Neto R, Forones NM (2016). HER2 expression as a prognostic factor in metastatic gastric cancer. Arq Gastroenterol, 53, 62-67.

Allgayer H, Babic R, Gruetzner KU, et al (2000). c-erbB-2 is of independent prognostic relevance in gastric cancer and is associated with the expression of tumor-associated protease systems. J Clin Oncol, 18, 2201-9.

Azarhoosh R, Ebneghasem R, Besharat S (2017). HER-2/ neu gene amplification in gastric adenocarcinoma and its relationship with clinical and pathological findings. $J$ Gastrointest Oncol, 8, 1046-50.

Bang YJ, Van Cutsem E, Feyereislova A, et al (2000). Trastuzumab in combination with chemotherapy versus chemotherapy alone for treatment of HER2-positive advanced gastric or gastro-oesophageal junction cancer (ToGA): A phase 3, open-label, randomized controlled trial. Lancet, 376, 687-97.

Begnami MD, Nonogaki S, Montagnini AL, et al (2011). Prognostic implications of altered human epidermal growth factor receptors (HERs) in gastric carcinomas: HER2 and HER3 are predictors of poor outcome. J Clin Onco, 29, 3030-36.

Bilici A (2014). Treatment options in patients with metastatic gastric cancer: current status and future perspectives. World $J$ Gastroenterol, 20, 3905-15.

Chao He, Xue-Yi Bian, Xing-Zhi Ni, et al (2013). Correlation of human epidermal growth factor receptor 2 expression with clinicopathological characteristics and prognosis in gastric cancer. World J Gastroenterol, 19, 2171-8.

Chua TC, Merrett ND (2012). Clinicopathologic factors associated with HER2-positive gastric cancer and its impact on survival outcomes - a systematic review. Int J Cancer, 130, 2845-56.

Cunningham D, Starling N, Rao S, et al (2008). Capecitabine and oxaliplatin for advanced esophagogastric cancer. N Engl J Med, 358, 36-46.

Dikshit R, Prakash CG, Chinthanie R, et al (2012). Cancer mortality in India: a nationally representative survey. Lancet, 379, 1807-16.

Fan XS, Chen JY, Li CF, et al (2013). Differences in HER2 over-expression between proximal and distal gastric cancers in the Chinese population. World J Gastroenterol, 19, 3316-23.

Ferdinando De Vita, Michele O, Alessio F, Maria M, Jole V,Beatrice Savastano (2014). Incidence and prognostic significance of HER2 overexpression in gastric cancer (GC): A mono institutional retrospective analysis. J Clin Oncol, 32, 160-6.

Ferlay J, Soerjomataam I, Ervik M, et al (2013). GLOBOCON 2012 v1.0, Cancer Incidence and Mortality Worldwide: IARC Cancer Base No. 11. Lyon, France: International Agency for Research on Cancer, 2013.

GLOBOCON Project (2015). Estimated Cancer Incidence, Mortality and Prevalence Worldwide in 2012. IARC (International Agency for Research on Cancer) /WHO. [Cited 8 Dec 2015].

Gravaalos C, Jimeno A (2008). HER2 in gastric cancer. A new prognostic factor and a novel therapeutic targt. Ann Oncol, 19, 1523-9.
Gupta P, Rao S, Bhalla S (2016). Human epidermal growth factor receptor 2 expression in gastric carcinoma and its association with histopathological parameters in Indian population. Indian J Cancer, 53, 505-11.

Hofmann M, Stoss O, Shi D, et al (2008). Assessment of a HER2 scoring system for gastric cancer: Results from a validation study. Histopathology, 52, 797-805.

ICMR (2016). Three Year Report of the Population Based Cancer Registries (PBCR) 2012-2014: Report of 27 PBCRs; National Cancer Registry Programme, Indian Council of Medical Research (ICMR), Bangalore; 2016.

Ishaky EA, El-Sharkawy SL, Ayob MM (2017). Immunohistochemical expression of HER-2/neu receptors in gastric carcinoma. J Arab Soc Med Res, 12, 13-18.

Kang YK, Yoon DH, Ryoo BY, Ryu MH (2010). Recent advances in chemotherapy for advanced gastric cancer. Asia Pac $J$ Oncol Hemato, 2, 67-74.

Kaur A, Dasnu CA (2011). Targeting the HER2 pathway for the therapy of lower esophageal and gastric adenocarcinoma. Exp Opin Pharmacother, 12, 2493-503.

Kunz PL, Mojtahed A, Ford JM, et al (2012). HER2 expression in gastric and gastroesophageal junction adenocarcinoma in a US population: clinicopathologic analysis with proposed approach to HER2 assessment. Appl Immunohistochem Mol Morphol, 20, 13-24.

Laboissiere RS, Rocha RM, Cabra MMDA, et al (2015). Association between HER2 status in gastric cancer and clinicopathological features: a retrospective study using whole-tissue sections. BMC Gastroenterol, 15, 157-62.

Lei Y, Huang J, Zhao Q, et al (2017). The clinicopathological parameters and prognostic significance of HER2 expression in gastric cancer patients: a meta-analysis of literature. World J Surg Oncol, 15, 1-7.

Maressch J, Schoppmann SF, Thallinger CM, Zielinski CC, Hejna M (2012). Her2/neu gene amplification and over-expression in stomach and esophageal adenocarcinoma: From pathology to treatment. Crit Rev Oncol Hematol, 82, 310-22.

Msika S, Benhamiche AM, Jouve JL, Rat P, Faivre J (2000). Prognostic factors after curative resection for gastric cancer. A population-based study. Eur J Cancer, 36, 390-6.

Nirmala P, Jing-shu Geng, Wencai LI, et al (2017). Human epidermal growth factor receptor 2 status of gastric cancer patients in Asia: results from a large, multicountry study. Asia Pac J Clin Oncol, 13, 249-60.

Park DI, Yun JW, Oh SJ, et al (2006). HER-2/neu amplification is an independent prognostic factor in gastric cancer. Dig Dis Sci, 51, 1371-9.

Pathmanathan R, Ho Kean F, Foo Yoke C (2018). Prevalence of HER2 positivity and its clinicopathological correlation in locally advanced/metastatic gastric cancer patients in Malaysia. J Gastrointest Canc, 49, 150-7.

Rahman R, Asombang AW, Ibdah JA (2014). Characteristics of gastric cancer in Asia. World J Gastroenterol, 20, 4483-90.

Rajagopal I, Niveditha SR, Sahadev R, et al (2015). HER2 expresion in gastric and gastroexophageal junction adenocarcinomas. J Clin Diagn Res, 9, 34-9.

Reichelt U, Duesedau P, Quaas A, et al (2007). Frequent homogeneous HER-2 amplification in primary and metastatic adenocarcinoma of the esophagus. Mod Pathol, 20, 120-9.

Rüschoff J, Hanna W, Bilous M, et al (2012). HER2 testing in gastric cancer: a practical approach. Mod Pathol, 25, 637-50.

Shabbir A, Qureshi MA, Khalid AB, et al (2018). Gastric adenocarcinoma expressing human epidermal growth factor receptor in South Asian population. Saudi J Gastroenterol, 24, 289-93. 
Shan L, Ying J, Lu N, et al (2013). HER2 expression and relevant clinicopathological features in gastric and gastroesophageal junction adenocarcinoma in a Chinese population. Diagn Pathol, 8, 76-82.

Son HS, Shin Ym, Park KK, et al (2014). Correlation between HER2 overexpression and clinicopathological characteristics in gastric cancer patients who have undergone curative resection. $J$ Gastric Cancer, 14, 180-6.

Van Cutsem E, Moiseyenko VM, Tjulandin S, et al (2006). Phase III study of docetaxel and cisplatin plus fluorouracil compared with cisplatin and fluorouracil as first-line therapy for advanced gastric cancer: A report of the V325 Study Group. J Clin Oncol, 24, 4991-7.

Van CE, Bang YJ, Feng-Yi R, et al (2015). HER2 screening data from ToGA: targeting HER2 in gastric and gastroesophageal junction cancer. Gastric Cancer, 18, 476-84.

Xiaowen Ge, Haixing W, Haiying ZBS (2015). Clinical significance of assessing HER2/neu expression in gastric cancer with dual tumour tissue paraffin blocks. Hum Pathol, 46, 850-7.

Yan B, Yau EX, Bte Omar SS, et al (2010). A study of HER2 gene amplification and protein expression in gastric cancer. J Clin Pathol, 63, 839-42.

\section{c) (i) (8)}

This work is licensed under a Creative Commons AttributionNon Commercial 4.0 International License. 